\title{
Short-term Wind Power Prediction Based on Particle Filter and Radial Basis Function Neural Network
}

\author{
Yongxiang WANG ${ }^{1, a}$, Guochu $\mathrm{CHEN}^{1, b}$ \\ ${ }^{1}$ College of Electrical Engineering, Shanghai Dianji University, Shanghai, 200240, China \\ aemail: 304611081@qq.com, bemail: chengc@sdju.edu.cn
}

Keywords: Particle Filter; RBF; Prediction Model; Wind Power; Renewable Energy

\begin{abstract}
In order to improve the accuracy of wind power prediction, this paper proposed a new short-term wind power prediction combined method based on particle filter and radial basis function neural network. First, the historical wind speed data are processed with particle filter, and the processed wind speed data combined the historical wind direction data and temperature data are using as the input data of the forecast model. Then, the PF-RBF neural network of wind power output forecasting model is established according to the new input data. The experimental results show that the proposed forecasting model has a good accuracy for wind power prediction.
\end{abstract}

\section{Introduction}

Nowadays, with the gradual consumption of coal, oil, and other nonrenewable fossil energy, the development of renewable energy has been widespread concerned in the world. As an important part of renewable energy, wind power is a kind of wide distribution, large reserves, and clean energy, and its development and utilization has been paid the world's attention [1-2]. Meanwhile, the wind power prediction has become a research hotspot. When large-scale wind power is connected to the grid, the instability of the wind power will seriously damage to the stable operation of power system and the power quality [3]. Thus, improving the accurate prediction of wind speed or wind power has an important practical significance on the stable operation of the power system and on reducing the operation cost of power system. During the wind power prediction development process, many methods were produced, such as Auto-Regressive and Moving Average Model [4], Kalman Filtering Method [5], and the method of Artificial Neural Network [6], Support Vector Machine [7] and so on, and these methods had good prediction effect on wind power prediction. However, in recent years some scholars have put forward some combination methods of wind power prediction, which can greatly improve the prediction accuracy in a certain extent. Wang et al. [8] proposing one combined method based on the improved empirical mode decomposition algorithm and neural network, comparing to the single forecast model, and getting a very good prediction effect. Chen et al. [9] made the information fusion technology apply to the wind power prediction, and proposed a wind power forecasting combined method by using cross entropy theory, effectively improving the prediction accuracy.

However, we found that the wind speed is often the main reason affecting the power output. The wind speed data getting from the wind farm are often poor regularity, which are usually with bad data. To solve this problem, the paper used particle filter for data treatment, proposing a new combined method based on particle filter and radial basis function neural network. The historical wind speed data are first smoothly processed by the particle filter, and the singular points from the wind turbine operation can be removed. Then, according to the processed wind data, historical wind direction and temperature, we established the wind power prediction model, which is based on the radial basis function neural network. Finally, the prediction results are compared with the actual wind power through the MATLAB platform for experimental simulation. 


\section{Particle Filter Algorithm and Realization in Wind Speed}

The idea of Particle Filter (PF) [10] is a Monte Carlo simulation method, based on principle of Bayesian estimation using a series particle to represent a probability, and it can be commendably used for treatment of nonlinear systems [11]. Historical wind speed data can be described as a series of volatility discrete point set, and through the state space model of the system, particle filtering can make full use of the state values at $k$-1combined with the present observations to process values at time $k$. Particle filter is established on the basis of Bayesian estimation, which is mainly to construct the posterior probability density function of the unknown system states by prior knowledge and the actual observation data. The state space model can be written as

$$
\begin{array}{ll}
\text { Process equation: } \quad x_{k}=f\left(x_{k-1}, u_{k-1}\right) \\
\text { Observation equation: } \quad y_{k}=h\left(x_{k}, v_{k}\right)
\end{array}
$$

where $f\left(\right.$ )and $h()$ denote the known state function and observation function respectively; $x_{k}$ is a state of the system at time $k, u_{k}$ and $v_{k}$ represents random noise vectors of given distributions; $y_{k}$ represents observation value at time $k, u_{k}$ and $v_{k}$ are independent of each other and independent of the past and current state.

The particle filter working principle can be described as the formula (1) and (2). It updates a priori knowledge through the priori probability density and new coming observation value, and obtains the desired posterior probability density. Then, according to Bayesian estimation principle, the posterior probability density can be got by both prediction and update steps. Thus, formula (1) and (2) can be written as

$$
\left\{\begin{array}{c}
p\left(x_{k} \mid y_{1: k-1}\right)=\int P\left(x_{k} \mid x_{k-1}\right) p\left(x_{k-1} \mid y_{1: k-1}\right) d x_{k-1} \\
p\left(x_{k} \mid y_{1: k}\right)=\frac{p\left(y_{k} \mid x_{k}\right) p\left(x_{k} \mid y_{1: k-1}\right)}{p\left(y_{k} \mid y_{1: k-1}\right)}
\end{array}\right.
$$

where $p\left(x_{k} \mid y_{1: k-1}\right)$ represents the priori probability density, $p\left(x_{k} \mid y_{1: k}\right)$ denotes the desired posterior probability density, $p\left(x_{k} \mid x_{k-1}\right)$ and $p\left(y_{k} \mid x_{k}\right)$ can be got by (1) and (2).

However, due to integral problems in formula (3), it is difficult to gain the analytic solutions from (3). Thus, using the empirical distribution to approximate the probability density function of the state is a effective solution, which is based on Monte Carlo principle sampling $\mathrm{N}$ independent and identically distributed samples from the posterior probability density. Therefore, posterior probability density can be approximately described as

$$
p\left(x_{k} \mid y_{1: k}\right) \approx \frac{1}{N} \sum_{i=1}^{N} \delta\left(x_{k}-x_{k}^{i}\right)
$$

where $\delta(\cdot)$ denotes the Dirac-delta function.

Notice that $p\left(x_{k} \mid y_{1: k}\right)$ sometimes is multivariable, non-standard form and can't be written as combinations of analytic function. The sampling process is often difficult. Therefore, the important distribution function is often introduced in the sampling, which is easy to realize sampling. The important distribution function divided into the form can be written as

$$
q\left(x_{k} \mid y_{1: k}\right)=q\left(x_{k} \mid x_{0: k-1}, y_{1: k}\right) q\left(x_{k-1} \mid y_{1: k-1}\right)
$$

where $q\left(x_{k} \mid y_{1: k}\right)$ denotes the important distribution function. When the observation value had been gotten, the new data will be added into the old samples $x_{0: k-1}^{i} \sim q\left(x_{0: k-1} \mid y_{1: k}\right)$ and getting new samples. Then, $p\left(x_{k} \mid y_{1: k}\right)$ can be got by samples independent and identically distributed samples from $q\left(x_{k} \mid y_{1: k}\right)$ and calculate the weights of samples. Thus, posterior probability density can be written as

$$
p\left(x_{k} \mid y_{1: k}\right) \approx \frac{1}{N} \sum_{i=1}^{N} w_{k}^{i} \cdot \delta\left(x_{k}-x_{k}^{i}\right)
$$


where $w_{k}^{i}$ denotes the normalized weights.

The specific operation steps of particle filter algorithm can be described as follows

Step 1: Initialization $(k=0)$

For $\mathrm{i}=1,2, \ldots \mathrm{N}$, obtain $\mathrm{N}$ samples from $p\left(x_{0}\right)$, and set the weight, $w_{0}^{i}=1 / N$.

Step 2: Importance sampling

First, for $\mathrm{i}=1,2, \ldots \mathrm{N}$, the particle sample can be got from $x_{k}^{i} \sim q\left(x_{k} \mid x_{k-1}^{i}, y_{1: k}\right)$, and obtain samples at $\mathrm{K},\left\{x_{k}^{i}, i=1,2, \ldots, N\right\}$.

Then, when the observation values had been gotten, calculate every particle's weight, make that the $\hat{w}_{k}^{i}=p\left(y_{k} \mid x_{k}^{i}\right)$, and the formula of weight updating is described as

$$
\hat{w}_{k}^{i}=\hat{w}_{k-1}^{i} \frac{p\left(y_{k} \mid x_{k}^{i}\right) p\left(x_{k}^{i} \mid x_{k-1}^{i}\right)}{q\left(x_{k}^{i} \mid x_{k-1}^{i}, y_{1: k}\right)}
$$

Then, normalization of the weight by

$$
w_{k}^{i}=\frac{\hat{w}_{k}^{i}}{\sum_{i=1}^{N} \hat{w}_{k}^{i}}
$$

Step 3: To determine whether resample

If $N_{\text {eff }}=1 / \sum_{i=1}^{N}\left(\hat{w}_{k}^{i}\right)^{2} \leq N_{T}$, go to Step 4; else go to Step 5. where $N_{\text {eff }}$ denotes the effective particle number.

Step 4: Resample

Sampling new particle according to the size of the weights, make it meet as $p\left(x_{k}^{\hat{i}}=x_{k}^{i}\right)=w_{k}^{i}$ and set $x_{k}^{i}=x_{k}^{\hat{i}}, w_{k}^{i}=1 / N . x_{k}^{\hat{i}}$ denotes the new particle.

Step 5: Output of state estimation can be written as

$$
\hat{x}_{k}=\sum_{i=1}^{N} w_{k}^{i} x_{k}^{i}
$$

Step 6: $k=k+1$, return to Step 2 .

Notice that wind speed is the main factor and it makes wind turbines generate power. The accuracy of historical wind speed data provided by the numerical weather forecast often directly decides the reliability prediction results of the final power. The particle filter processing on the historical wind speed can better improve the accuracy of forecasting system. In this article, the experimental data of wind speed are based on 30 days data of the northeast wind farm of China. Data is from 30 days totally 720 sets. Both the process noise and observation noise are in the set 1 , and the number of sample particles is 200 . It can be seen the processed wind speed is coincided with the actual value, which can be seen from Figure 1.

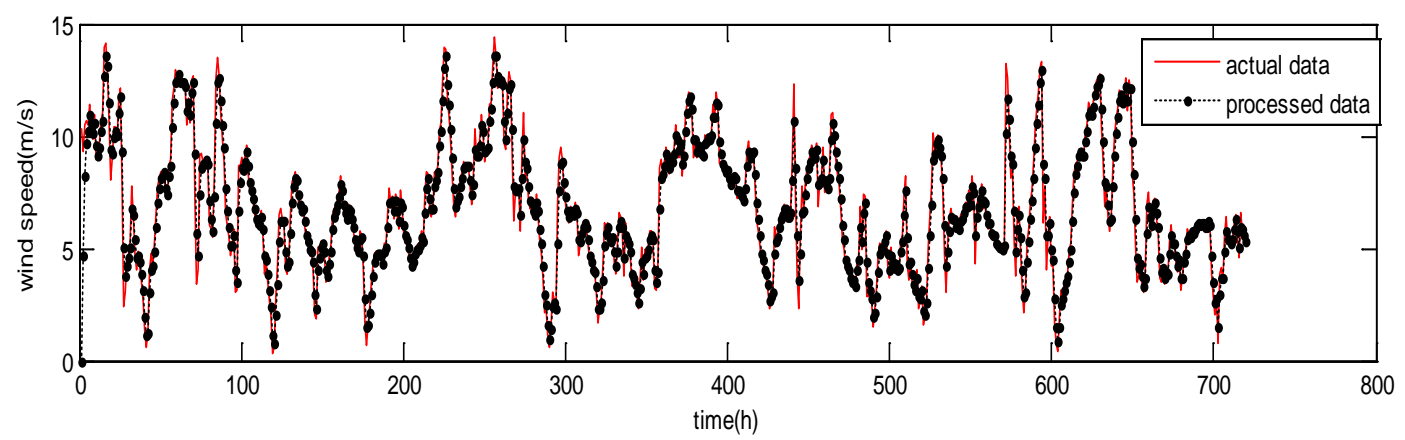

Fig.1. Time series of wind speed and processed wind speed 


\section{Particle Filter RBF neural network wind power prediction model}

Radial basis function (RBF) neural network is a kind of optimal feed forward network, which has strong input ability and complete mapping ability. Compared with other feed forward network, RBF network has the advantages of simple structure, simple training, and good local approximation performance and few parameters to set and so on. Thus, it is widely used in nonlinear optimization, time series prediction, pattern recognition and scientific fields and so on [12].

Notice that the main factor of wind turbines generating power is wind speed and its data collected by wind plant often show a random fluctuation vibration characteristic. Thus, the historical wind speed smoothly processed by particle filter can make the regularity of data be further enhanced, and the processed data are used as the new input of the network model. Then, the PF-RBF neural network model is established, where the input are the new wind speed data, historical wind direction and historical temperature. And these data are all normalized in this paper. Finally, the wind power is output through the prediction model. The process of the prediction model is shown in figure 2.

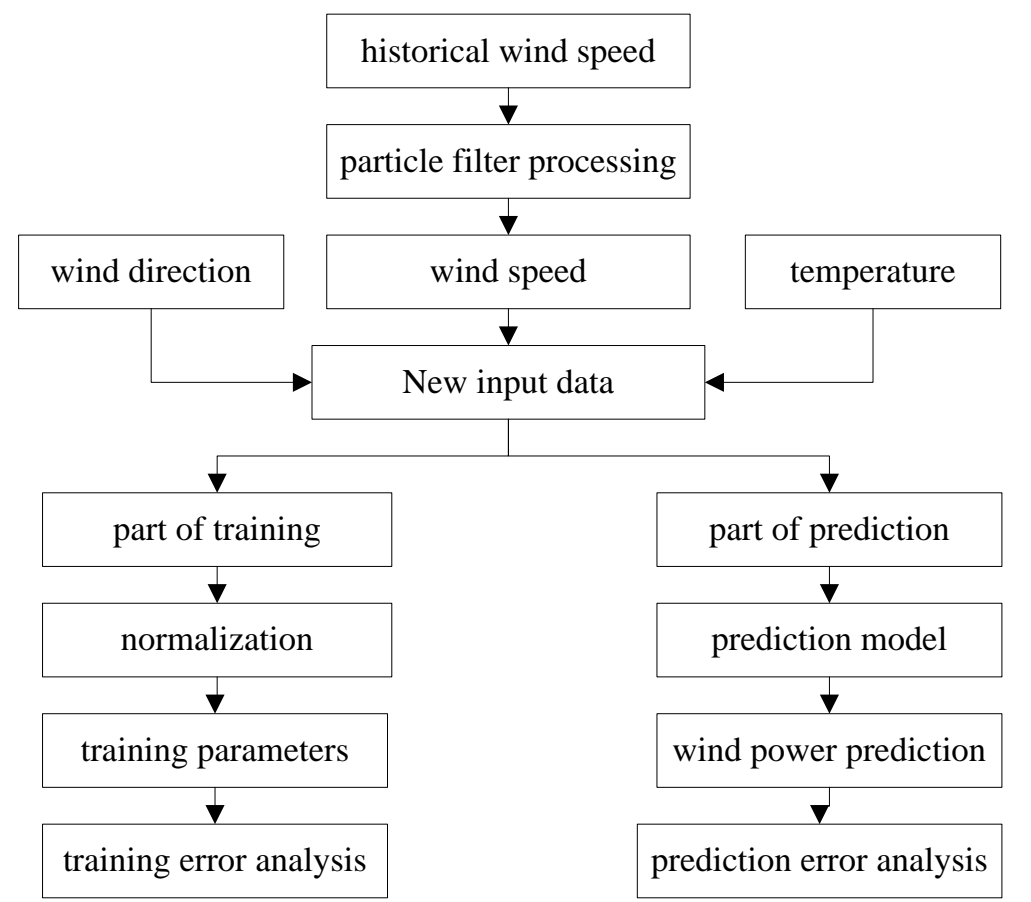

Fig. 2. Wind power prediction model based on PF-RBF neural network

The wind power prediction based on PF-RBF neural network steps are as follows:

Step1: Filter processing: the historical wind speed data are smoothly processed by particle filter, through the wind speed at time $k-1$ combined with the new arrival value wind speed to forecast state at time $k$, and produce a series of strong regularity of wind data.

Step2: Normalizing data: make the processed wind speed data with historical wind direction and historical temperature data as new input vectors of the model, and use the normalized formula, which is designed as

$$
T=T_{\text {min }}+\frac{T_{\text {max }}-T_{\text {min }}}{X_{\text {max }}-X_{\text {min }}}\left(X-X_{\text {min }}\right)
$$

where $T$ denotes the output of the target; $X$ represents the new input data of the model; $X_{\max }$ and $X_{\min }$ are respectively the maximum value and minimum of the new input data; the maximum and minimum values of the target output are set as $T_{\max }=1, T_{\min }=-1$.

Step3: Training the network: according to the mapping relationship between each layer established by radial basis function neural network, the network is trained to determine the parameters of the network, then establish network prediction model.

Step4: Neural network prediction: enter the new normalized wind power data in the trained 
network, obtain the prediction value of the network.

Step5: Anti-normalization: anti-normalized the output data by formula (11), and calculate the wind power value.

$$
X=X_{\text {min }}+\frac{X_{\text {max }}-X_{\min }}{T_{\text {max }}-T_{\text {min }}}\left(T-T_{\min }\right)
$$

Step6: Prediction analysis: make the actual wind power and forecasting power show in the chart, and calculate the prediction error.

\section{Simulation and analysis}

In order to verify the validity of the prediction model, the experimental data of wind speed are based on 30 days data of the northeast wind farm in June 2007. Record a group of data per hour, with a total of 720 groups. All wind speed data are processed by the particle filter algorithm, and the processed wind speed result is shown in Figure 1. Then the singular points in the process of filtering and non-normal operation point were removed from the data, remaining 697 groups of data. In the experiment, select the first 577 groups of data as training data of the network, and the last 120 sets of data are as test samples for power prediction in future one hour. Through the MATLAB simulation platform to predict, the experimental results are as shown in figure 3.

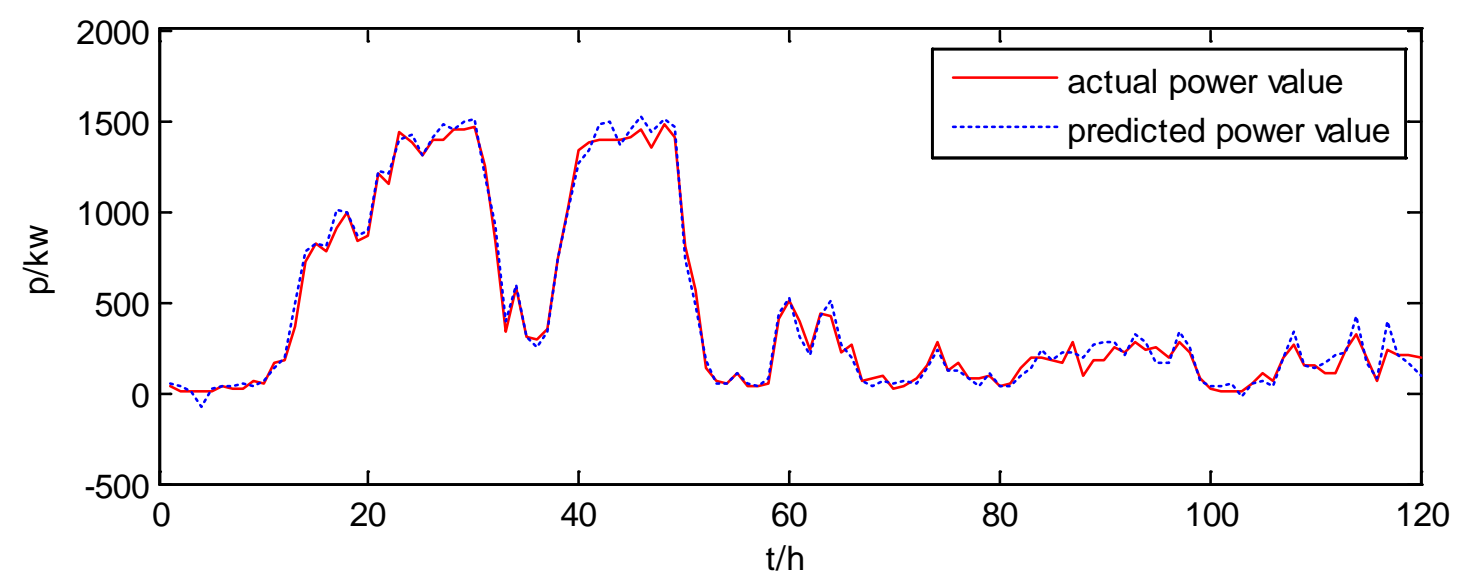

Fig.3. Comparison of PF-RBF prediction value and the actual measured value in $120 \mathrm{~h}$

The experiment use the Mean Absolute Percentage Error (MAPE) evaluate the accuracy of the prediction model, and the error formula can be described as

$$
M A P E=\frac{1}{N} \sum_{t=1}^{N}|Y(t)-T(t)| \times 100 \%
$$

where $T(t)$ denotes the actual value at time $t ; Y(t)$ is the predictive value at time $t$; $N$ represents the number of test samples.

Through Matlab simulation platform, and calculated by the formula (12) for 120 hours prediction, the mean absolute percentage error is $8.04 \%$. Besides, to further validate accuracy range of this prediction method, through many simulation experiments, respectively, calculate continuous 48, 72 , and 96 hours prediction error, and the experimental results as shown in Table 1.Through the treatment of the wind speed, the mean absolute percentage error of PF-RBF model is less than the previous single RBF neural network model, the prediction accuracy has much good improvement. 
Tab.1 Comparison MAPE of different hours of PF-RBF and RBF model

\begin{tabular}{|c|c|c|}
\hline \multirow{2}{*}{ Prediction Hours (h) } & \multicolumn{2}{|c|}{ MAPE (\%) } \\
\cline { 2 - 3 } & PF-RBF Model & Single RBF Model \\
\hline 48 & 6.73 & 12.19 \\
\hline 72 & 7.58 & 12.42 \\
\hline 96 & 7.95 & 12.48 \\
\hline
\end{tabular}

\section{Conclusion}

The wind power prediction model has been proposed in this paper, which was based on particle filter and radial basis function neural network. The historical wind speed data smoothly processed by particle filter, which can make the regularity of the data be further enhanced. And it provided more reliable input data for the prediction model. Then the PF-RBF neural network prediction model was established, where the input are the processed wind speed data, historical wind direction and historical temperature. The data were trained by the normalized data on the RBF neural network, and then the test data are act as new input of the network for realizing power prediction. The parameter of the network was trained by using the normalized data and then inputting the test data in the network, which realized power of prediction. The simulation results have shown that the prediction method given in this paper has good predictive precision in short-term wind power prediction, which will provide significant practical information for wind power prediction and also ensure the grid operate safely.

\section{Acknowledgement}

In this paper, the research was sponsored by scientific research innovation projects of Shanghai municipal education commission (Grant No. 13YZ140) and the key disciplines of Shanghai Municipal Education Commission of China (Grant No. J51901).

\section{References}

[1] Yongqian Liu, Shuang Han, Yongshen Hu. Review on short-term wind power prediction [J]. Modern electric power.2007, 24 (5): 6-11

[2] Jin g Li, Jiahua Song, Weisheng Wang. Modeling and dynamic simulation of variable speed wind turbine with large capacity [J]. Proceedings of the CSEE. 2004, 24(6):100-105

[3] BERNH ARD E, BRET T O, MARK L A, et al. Predicting the wind [ J] . IEEE Power \& Energy Magazine, 2007, 5( 6) : 78-89

[4] Kamal L, Jafri Y Z. Time series models to simulate and forecast hourly averaged wind speed in Quetta, Pakistan [J]. Solar Energy, 1997, 61(1): 23-32

[5] Bossanyi E A. Short-term wind prediction using kalman filters [J]. Wind Engineering,1985, 9(1): 1-8

[6] Gaofeng Fan, Weisheng Wang, Chun Liu, Huizhu Dai. Wind power prediction based on artificial neural network [J]. Proceedings of the CSEE, 2008, 28(34): 118-123

[7] Shuangbin Qi, Weiqing Wang, Xinyan Zhang. Wind speed and wind power prediction based on SVM [J].East China Electric Power, 2009, 37(9): 1600-1603

[8] Peng Wang, Guochu Chen, Yufa Xu, Jinshou Yu. Improved empirical model decomposition and its application to wind power forecasting [J]. Control Engineering of China, 2011, 4(18):588-591

[9] Ning Chen, Qian Sha, Yi Tang, Lingzhi Zhu. A combination method for wind power predication based on cross entropy theory [J]. Proceedings of the CSEE, 2012, 32(4): 29-34 
[10]Jayesh H K, Petar M D. Gaussian sum particle filtering[J]. IEEE Transactions on signal processing, 2003, 51(10):2602-2612

[11] Yue Luo. Research on remaining useful life prediction of lithium-ion battery with particle filter [D].Harbin: Harbin institute of technology. 2012, 27-28

[12] Yaoyao He, Qifa Xu, Shanlin Yang. A power load probability density forecasting method based on RBF neural network quantile regression [J]. Proceedings of the CSEE.2013,33(1): 93-98 